\title{
Contributions to the Joint DFG-RSF Project - Generation of Ultra-Short Microwave Pulses -
}

\author{
$\underline{\text { A. Marek }}^{1}$, K. A. Avramidis ${ }^{1}$, S. M. Copplestone ${ }^{2}$, N. S. Ginzburg ${ }^{3}$, S. Illy ${ }^{1}$, J. Jelonnek ${ }^{1,4}$, \\ J. Jin ${ }^{1}$, S. V. Mishakin ${ }^{3}$, P. Ortwein ${ }^{2}$ and M. Thumm ${ }^{1,4}$
}

${ }^{1}$ IHM, ${ }^{4}$ IHE, Karlsruhe Institute of Technology (KIT), Karlsruhe, Germany, alexander.marek@kit.edu

${ }^{2}$ Institute of Aerodynamics and Gas Dynamics, University of Stuttgart, Stuttgart, Germany

${ }^{3}$ Institute of Applied Physics, Russian Academy of Sciences (IAP- RAS), Nizhny Novgorod, Russia

\section{Introduction}

In a joined DFG-RSF project of the Institute of Applied Physics (IAP-RAS) and the Institute for Pulsed Power and Microwave Technology (IHMKIT), the generation of a periodic sequence of powerful short RF pulses is studied [1]. Such powerful pulses in the millimeter and sub-millimeter wavelength range can be useful for a large number of fundamental problems and practical applications, including diagnostics of plasmas, photochemistry, biophysics, new locating systems, and spectroscopy of various media.

A periodic sequence of powerful pulses can be generated in a feedback loop consisting of an amplifier and a saturable absorber [1]. The saturable absorber acts as a nonlinear filter which transmits high intensity signals while signals with low intensity are absorbed. In such a feedback loop, the periodic signal is generated by the mechanism of passive mode-locking. In the millimeter wavelength range, gyro-devices as the gyro-traveling-wave-tube (gyro-TWT) are well suited for the realization of both devices, the amplifier and the nonlinear absorber.

\section{Components of a feedback loop}

Amplifier and absorber can be realized as gyroTWTs with helical interaction region [2]. These devices provide a series of advantages compared to classical gyro-TWTs based on cylindrical interaction circuits with dielectric losses. The helical gyro-TWTs can operate at the $2^{\text {th }}$ cyclotron harmonic and therefore, the required magnetic field is reduced by the factor of 2 compared to classical gyro-TWTs operating at the fundamental cyclotron harmonic. Further advantages are that helical gyro-TWTs offer a broad frequency bandwidth up to $20 \%$, which is important for the generation of ultra-short pulses in the subnanosecond range, and that they are not sensitive to electron velocity spread. Also, it is possible to operate helical them with a single window for the input and output of the RF signal [2]. In the single input/output window operation it is possible to couple even highpower signals into the device, which is an essential condition in the feedback loop. In addition, the single input/output window simplifies the coupling of the amplifier and the absorber in a feedback loop.

Amplifier and absorber will operate in different operating regimes. The amplifier will run in a regime optimal for the maximal amplification of ultra-short pulses [3], while the absorber will run in the so-called Kompfner dip regime [4].

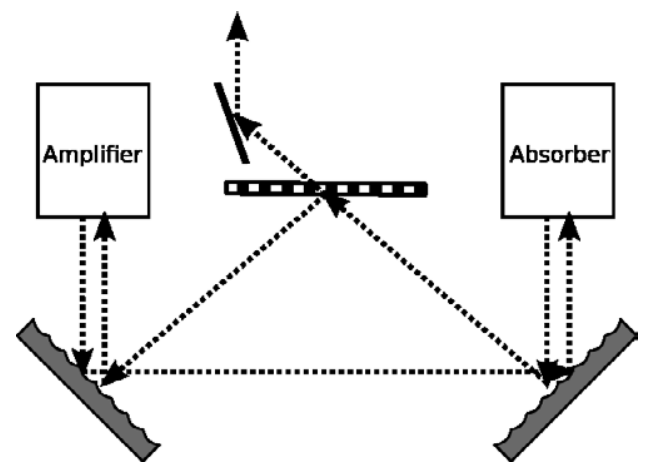

Fig. 1. Amplifier and absorber devices working with single input-output window coupled with a quasi-optical mirror system.

For an optimum amplification of ultra-short pulses, the group velocity in the helical section of the amplifier is higher than the axial velocity of the electron beam. Therefore, the RF pulse "slips" over the electron beam and accumulates energy from different fractions of the beam [3].

The absorber is designed similar to a standard helical broadband amplifier, i.e. the group velocity and the axial velocity of the electron beam should be equal over a broad frequency bandwidth. The Kompfner dip regime then is realized by a detuning of the magnetic field and the electromagnetic wave is attenuated by the electron beam.

In an experimental setup, the coupling of the two gyro-devices can be realized by a quasi-optical mirror system. To decouple a fraction of the signal oscillating in the feedback loop, a semi-transparent mirror is required. Such a semi-transparent mirror can be realized as polarizing beam-splitter based on a stack of rectangular metal plates as described in [5]. For optimum operation, the decoupling should take place only on the signal trail from the absorber back to the amplifier. Therefore, a separation of the signal trails amplifier to absorber and absorber to amplifier is necessary. For this purpose, we can take advantage of an attribute of helical gyro-TWTs in the single window operation: The output signal is cross-polarized to the input signal. As result, mirrors with sinusoidal grating can be used as effective polarization splitter. A possible quasi-optical coupling system based on the previous described technics is shown in figure 1 .

\section{Simulations}

Full-wave PIC simulations including the interaction between electrons and electromagnetic waves are 


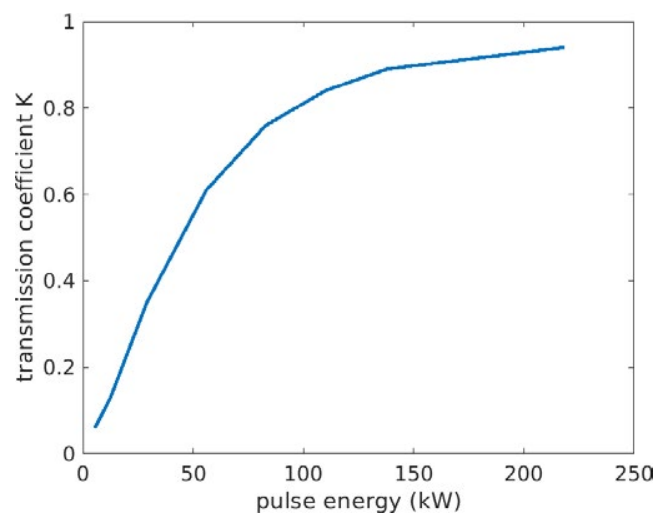

Fig. 2. Dependence of the transmission coefficient on the power of the incident signal for a helical gyro-TWT operating in the Kompfner dip regime.

performed with the advanced simulation program PICLas [6], developed by the Institute of Aerodynamics and Gas Dynamics (IAG) at the University of Stuttgart. The required high-order hexahedral meshes are generated with the mesh-generator HOPR [7], also developed by the IAG.

In the following, simulations of an absorber as suggested in [8] for a feedback loop at a center frequency of $30 \mathrm{GHz}$ are performed. The suggested feedback loop is designed to provide pulses with a peak-power up to $400 \mathrm{~kW}$ and a width of only $0.25 \mathrm{~ns}$ by a separation of 5-10 ns [8]. The absorber has a mean waveguide radius of $0.4 \mathrm{~cm}$ with a corrugation of amplitude $0.1 \mathrm{~cm}$ and a period of $1.0 \mathrm{~cm}$. The electron beam has a beam voltage of $54 \mathrm{kV}$, a current of $2.4 \mathrm{~A}$ and a pitch factor of 0.53 .
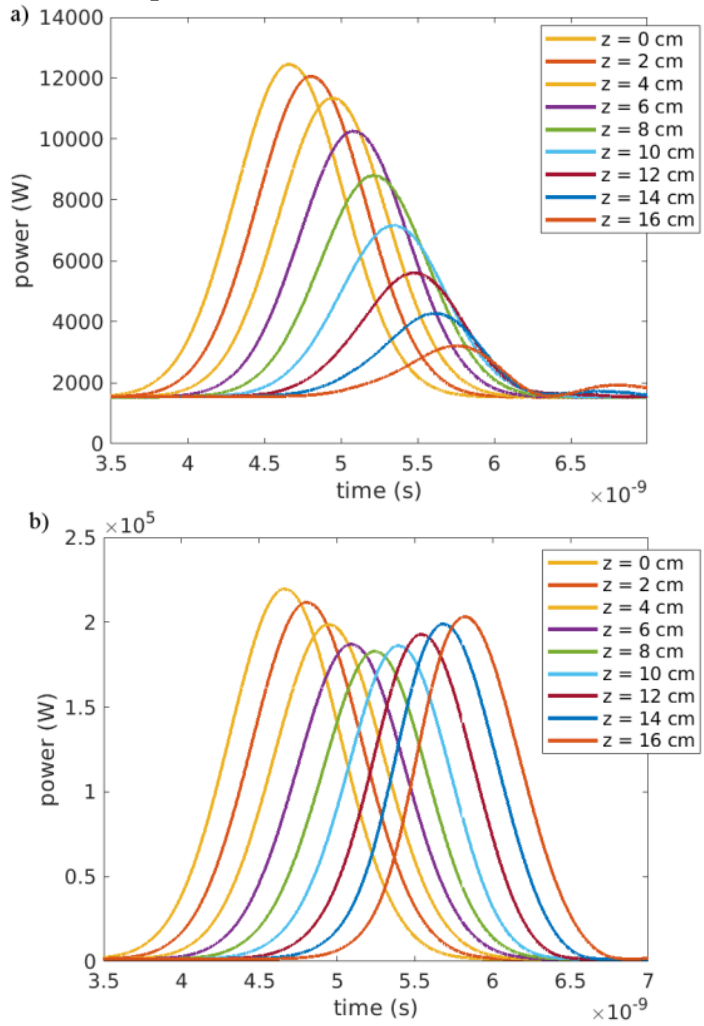

Fig. 3. RF-power of a Gaussian pulse propagating along the interaction region of a helical gyro-TWT operating in the Kompfner dip regime, for a low power input signal a), and a high power signal $b$ ).
In figure 2 , the simulated transmission coefficient for short Gaussian pulses with different peak powers is shown. While weak signals are almost completely absorbed, high power signals are only weakly attenuated. Therefore, the simulations proof the qualification of gyro-TWTs operating in the Kompfner dip regime as saturable absorbers. From figure 3, the saturable absorber effect can be understood in detail. In figure $3 \mathrm{a}$ ), the propagation of a $10 \mathrm{~kW}$ Gaussian RF pulse along the absorber is shown. The absorption of almost the complete pulse can be clearly seen. In figure $3 \mathrm{~b}$ ), the propagation of a high-power input pulse (200 kW) along the same absorber is shown. It can be seen that first a maximum fraction of $30 \mathrm{~kW}$ of the input power is absorbed by the electron beam. The transfer of the RF power to the kinetic energy of the electrons causes a shift from the Kompfner dip regime to the amplification regime. Thus, the RF pulse gains a fraction of its previous emitted energy back. A comparison of the input and output signal shows that the high-power pulse lost only a small fraction of its power. Therefore, the device exhibits properties of a saturable absorber.

\section{Acknowledgements}

The research is supported by the joint RSF-DFG project (Je 711/1-1, 16-42-01078) "Generation of U1trashort Pulses in Millimeter and Submillimeter Bands for Spectroscopy and Diagnostic of Various Media Based on Passive Mode-locking in Electronic Devices with Nonlinear Cyclotron Absorber in the Feedback Loop".

\section{References}

1. Ginzburg, N. S., et al. "Generation of a periodic sequence of powerful ultrashort pulses in a traveling wave tube with bleachable absorber in the feedback loop." Technical Physics Letters 41.9 (2015): 836-839.

2. Denisov, Gregory G., et al. "Microwave system for feeding and extracting power to and from a gyrotron traveling-wave tube through one window." IEEE Electron Device Letters 35.7 (2014): 789-791.

3. Ginzburg, N. S., et al. "Mechanisms of amplification of ultrashort electromagnetic pulses in gyrotron traveling wave tube with helically corrugated waveguide." Physics of Plasmas 22.11 (2015): 113111.

4. Ginzburg, N. S., et al. "Generation of trains of ultrashort microwave pulses by two coupled helical gyro-TWTs operating in regimes of amplification and nonlinear absorption." Physics of Plasmas 24.2 (2017): 023103.

5. Mendis, Rajind, et al. "Artificial dielectric polarizing-beamsplitter and isolator for the terahertz region." Scientific Reports 7.1 (2017): 5909.

6. Munz, Claus-Dieter, et al. "Coupled particle-in-cell and direct simulation Monte Carlo method for simulating reactive plasma flows." Comptes Rendus Mécanique 342.10-11 (2014): 662-670.

7. Hindenlang, Florian, Thomas Bolemann, and C-D. Munz. "Mesh curving techniques for high order discontinuous Galerkin simulations." IDIHOM: Industrialization of high-order methods-a top-down approach. Springer, Cham, 2015. 133-152.

8. Ginzburg, Naum S., et al. "Ultrawideband Millimeter-Wave Oscillators Based on Two Coupled Gyro-TWTs With Helical Waveguide." IEEE Transactions on Electron Devices 65.6 (2018): 2334-2339. 